\title{
Challenges in Feeding Children Posed by the COVID-19 Pandemic: a Systematic Review of Changes in Dietary Intake Combined with a Dietitian's Perspective
}

\author{
Heather Campbell ${ }^{1}$ - Alexis C. Wood ${ }^{2}$ \\ Published online: 15 May 2021 \\ (C) The Author(s), under exclusive licence to Springer Science+Business Media, LLC, part of Springer Nature 2021
}

\begin{abstract}
Purpose of Review To examine the evidence that the dietary quality of children changed between the period preceding the COVID-19 pandemic and the first year during the pandemic.

Recent Findings A systematic review of the evidence for dietary changes occurring as a result of the pandemic-related restrictions, in Part I of this article, yielded 38 original research articles. These articles had conflicting results, some describing improvements in overall quality and some describing deteriorations. As a whole the studies were characterized by a low study quality, and children were poorly represented. Taken together, these studies do not provide enough evidence to draw conclusions about whether dietary habits changed or not as a result of the pandemic. However, in a wider, narrative review of the psychosocial changes occurring as a result of the COVID-19 pandemic, and the known associations of these factors with a dietary intake in Part II, we conclude that there is a reason to expect that the dietary quality of children might have been adversely affected by the COVID-19 pandemic.

Summary One the one hand, the literature fails to provide conclusive evidence on changes in the dietary quality of children resulting from the COVID-19 pandemic. On the other hand, the broader literature supports the hypothesis that children's dietary quality will have declined during the pandemic. Taken together, we urgently need more high-quality research on children's changes in dietary intake occurring over the pandemic. This will provide important information on whether any potential longterm consequences of such changes, if they exist, need to be examined and ameliorated.
\end{abstract}

Keywords COVID-19 $\cdot$ Diet quality $\cdot$ Systematic review $\cdot$ Disparities

\section{Introduction}

\section{Context}

In December 2019, the deep sequencing of samples from the lower respiratory tract of a cluster of patients presenting with viral pneumonia of no known cause in Wuhan, China, revealed the existence of a novel pathogen, named severe acute

This article is part of the Topical Collection on Maternal and Childhood Nutrition

Alexis C. Wood

LekkiWood@Gmail.com

Glory Nutrition, Birmingham, AL, USA

2 USDA/ARS Children's Nutrition Research Center, Baylor College of Medicine, 1000 Bates Street, Houston, TX 77071, USA respiratory syndrome coronavirus 2 (SARS-CoV-2). SARSCOV-2 causes the disease initially named as "2019 novel coronavirus" (2019-nCoV), more commonly now known as COVID-19 [1]. COVID-19 spread rapidly throughout the globe, leading the World Health Organization (WHO) to declare COVID-19 a global pandemic in March 2020 [2]. At the time of writing (February 2021), World0meter reported the cumulative global number of cases to be 107,639,508, resulting in 2,358,130 deaths $[3,4]$, while in the United States (US) The Centers for Disease Control (CDC) reported over 26,000 cumulative US cases resulting in 463,659 deaths [5]. The efforts of the public health community to understand and stem the spread of COVID-19 have been described as "unprecedented" [6]. As well as encouraging individual-level behaviors such as mask wearing, hand washing, and the use of hand sanitizer [7], there has been the widespread implementation of broader "lockdown" mandates which can include everything from relatively short periods of self-isolation/quarantine for those 
exposed to SARS-COV-19 or testing positive for COVID-19, to local, regional, and national restrictions on business openings, the opening of schools and places of worship, and restrictions on personal non-essential travel to distances as short as a one-mile radius from home. Since the declaration of COVID19 as a pandemic, almost 4 billion people, that is, nearly half the world's population, have been asked to participate in some form of confinement, be it a mandatory or recommended curfew, isolation, or quarantine, or some other forms of restriction on social contract (report cited in [8]). Based on this universal and sometimes extended restriction, the adverse effects of COVID-19 are not expected to be limited to symptoms and outcomes of COVID-19 alone, but are anticipated to include multiple other mental, physical, and psychosocial sequelae arising from the pandemic environment.

\section{Rationale}

Health pandemics have been noted to increase populationlevel mental distress, and the COVID-19 pandemic is no exception as starkly illustrated by observed and projected increases in the rates of dying by suicide [9-11]. Layered on top of this, data from previous disease outbreaks show that the effects of lockdowns, in all their forms, can have a dramatic impact on health behaviors. In addition to reports of reductions in physical activity [12-14] and increases in sedentary time [12,13] (although we note some reports to the contrary [15]), the separation from loved ones, the loss of freedom, and the uncertainty and boredom have also been associated with declines in those mental health factors which, in turn, influence health behaviors. A recent, scoping review found that a substantial number of studies before the current pandemic associated lockdowns with generally high psychological distress, high depressive symptoms, emotional exhaustion, detachment from others, irritability, insomnia, poor concentration, indecisiveness, fear, nervousness, sadness, deteriorating work performance, and reluctance to work/consideration of resignation, with those symptoms related to low mood and/ or irritability "standing out" as having a particularly high prevalence [16]. Data from around the globe suggest that the current COVID-19 pandemic is having similar adverse effects [17]. In addition, although data are scarce, it has been noted that women, especially those who are caregivers to young children, may be disproportionately affected by the psychosocial/psychological effects of the pandemic. Early reports suggest that violence against women is increasing [8]. Women may have more severe and more prevalent disturbances in sleep and psychological functioning [18] and a higher prevalence of mental distress $[19,20]$, as well as increased symptoms of anxiety [21], stress [21], and depression [21]. Even though not all data are consistent in reporting stronger effects on the mental health of women than men [22], it is clear that in most nations, women are bearing the majority of financial impacts resulting from the pandemic, such as increased job losses [23]. Globally, women still carry the greatest burden in child rearing [24], a disparity which may have increased further during the COVID-19 pandemic [25]. Therefore the adverse effects of the pandemic which are disproportionately affecting women-plus the effects of school closures and reduced social contacts-gives rise to the expectation of similar negative sequelae on children. Given that strong associations of dietary quality with factors such as good mental health [26, 27] and economic security [28] have been well established across women, children, and adolescents, it may be expected that one health behavior which will be adversely affected by the current pandemic will be maternal and child dietary quality.

\section{Objectives}

The purpose of this review is to combine the current evidence in the published literature on how the COVID-19 pandemic may have affected the dietary quality of families with the personal experience of one registered dietitian working with families during this period. In Part 1, we conduct a systematic review of dietary changes during the COVID-19 pandemic and summarize the key findings of this body of literature. The inconsistent findings and the methodological limitations in this literature serve to highlight that we know very little about how diets have changed over the COVID-19 pandemic, particularly in the case of children. In Part II, we share the observations and experiences one of the co-authors has gained through her work as a registered dietitian, practicing through this pandemic, and provide evidence-based hypotheses on the effects these will have on the dietary quality of families. This protocol was reviewed by the Institutional Review Board at Baylor College of Medicine (protocol number:H-49021), which determined that this activity does not constitute human subject research and so does not fall under the regulations for IRB review of human subject research.

\section{Part 1: Summary of the Literature}

\section{Methods}

\section{Initial Search}

The senior author extracted all manuscripts in MEDLINE resulting from the search terms "[COVID-19 OR SARSCOV-2] AND [Diet]" on February 10, $2021(N=508$; Fig. 1). Articles were selected for inclusion (Table 1) using pre-defined data fields representing each eligibility/exclusion criterion. The overall protocol was registered using the International Prospective Register of Systematic Reviews (PROSPERO [29]; registration number CRD42021160754) 


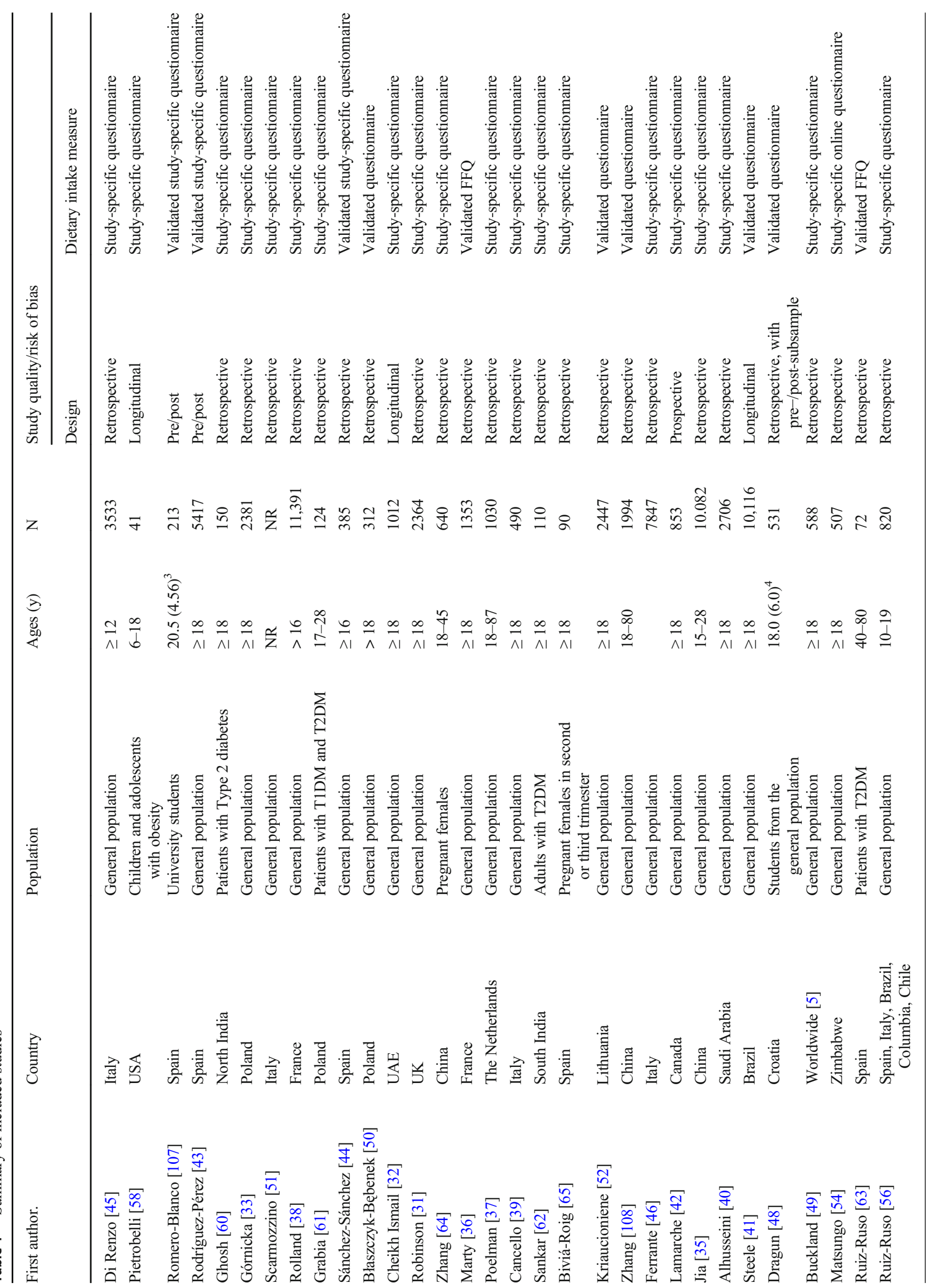


and reported according to the guidance in the statement on preferred reporting items for systematic reviews and metaanalyses (PRIMSA [30]).

\section{Eligibility Criteria}

Manuscripts were included which were available in English, contained primary data analysis, and reported on differences in the intake of specific foods before and after March 2020 (i.e., before and during the COVID-19 pandemic, in broad terms).

\section{Exclusion Criteria}

After excluding duplicates $(N=2)$, studies were sequentially excluded which did not have the full text available in English $(N=15)$, did not describe dietary changes occurring from before to during the COVID-19 pandemic $(N=383)$, did not include primary data $(N=36)$, did not report on changes in specific food groups but related behaviors (e.g., reported on eating habits, types of food, and/or appetitive traits; $N=29$ ), conducted data reduction efforts such that changes in individual foods or food groups could not be described (e.g., used principle component analysis; $N=2$ ), or reported only on the associations of individual characteristics with dietary changes and did not include an analysis of population-based change $(N=3)$ were excluded. This process yielded a final sample of 38 studies (Table 1).

\section{Data Extraction}

Information was extracted from each included study on (1) study characteristics (sample size, country of origin for participants), (2) study design, (3) ascertainment of sample, and (4) method of obtaining dietary intake and (5) results of analysis.

\section{Risk of Bias}

Study quality was assessed via (1) study design and (2) the method of measuring dietary intake (Table 2 ) and scored within a range of $10-8$. The majority of studies took a retrospective approach.

\section{Results}

\section{Description of Studies}

Study quality is generally low, ranging from zero to five out of a possible maximum score of seven, with a mean of study quality score of 2.11 (Fig. 2). The low assessment of study quality is driven by the use of cross-sectional designs which relied on retrospective reports of pre-pandemic dietary intake (79\% of studies) and by assessments of dietary intake prone to 


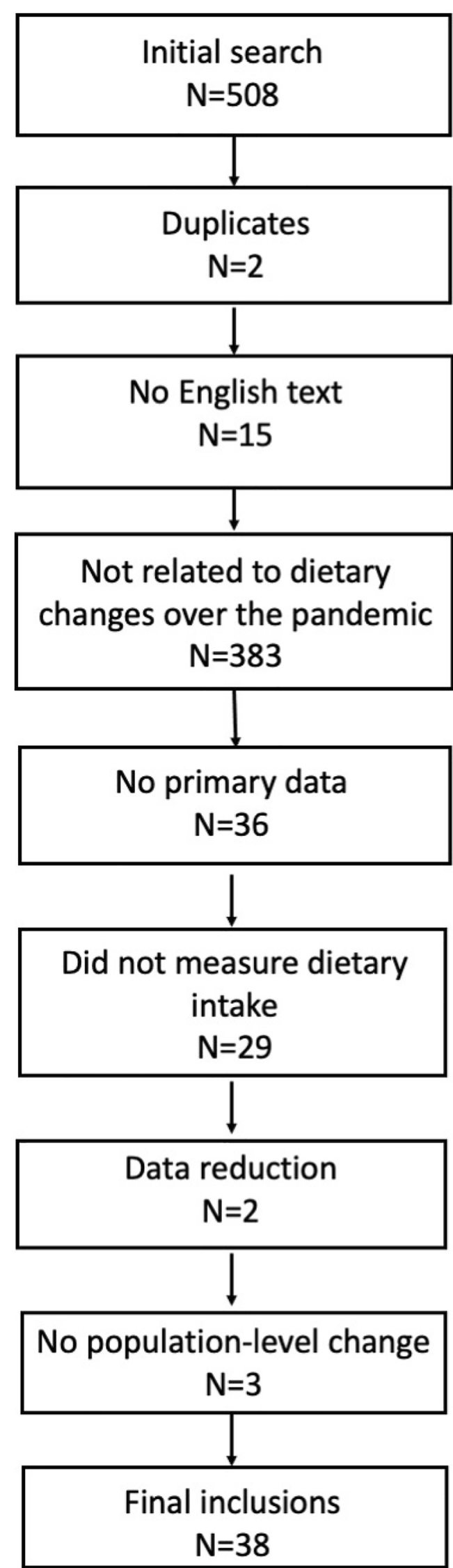

Fig. 1 Study selection bias: no studies used objective data or multiple 24-h recalls of intake (the gold standard self-report methodology). Only 13 studies $(34 \%)$ reported that their measure of dietary intake had been validated in previous analyses (Table 1 ).

A strength of the overall literature is the representation of a broad range of countries and cultures. However, within the impressive geographical breadth, the specific populations studied are limited. The majority of studies (74\%) reported on dietary changes in adults from the general population, with only six studies $(16 \%)$ in clinical populations, with four including patients with diabetes and two including pregnant females. A similarly small number of studies presented data on children or adolescents $(11 \%)$, although one additional study included children over the age of twelve in the overall analysis (Table 1).

\section{Dietary Patterns}

Overall, it is not clear whether people perceive their dietary quality to have changed, and if so in which direction, over the pandemic. Reports of a decline in overall dietary quality were available from studies based in the United Kingdom (UK) [31], United Arab Emirates (UAE) [32], Poland [33], Denmark [34], Japan [35], France [36], of no change in the Netherlands [37], and Italy [38]. Mixed results within a study were also available from Italy where one study reported both perceived improvements (34\%) and declines (19\%) [9]. Improvements in overall dietary quality were available from Saudi Arabia [40], Brazil [41], Canada [42], and Spain (via a closer adherence to a Mediterranean-style diet [43, 44]).

\section{Specific Foods}

When looking at data from adults, a consistent pattern of changes in the intake of individual foods, or food groups, could not be isolated from the literature. Changes suggesting an improvement in dietary quality are indicated by reports of increases in the intake of cereals [45] and whole grains [33, $42,46]$, nuts [47], pulses, beans and/or legumes [33, 36, 41, 42, 44-46, 48, 49], white meat [45], eggs [33, 47, 50], potatoes [50], fish and/or seafood [34, 36, 42, 44, 48], milk/other dairy [33, 42, 47], fruits, and/or vegetables [33, 36, 41-43, 46-48, 51-53]. Similar positive changes are also suggested by reports of decreases in refined grains [42], sugar sweetened beverages (SSBs) [32, 33, 43, 44], sweets [48], and red meat [52]. Yet some studies disputed these positive findings, reporting perceived increases in carbonated and SSBs [34, $36]$, and processed/red meats [33, 36, 43, 47] and decreases in the intakes of fish [33, 45, 47, 52], cereals/whole grains [33, $48,54]$, dairy [35, 48, 54], nuts [48, 54], legumes [34, 47], and fruits and/or vegetables [33-35, 54, 55]. Other changes associated with a decrease in dietary quality during the pandemic include reported increases in butter and margarine [44], 
Table 2 Criteria for assessing study quality

\begin{tabular}{|c|c|c|}
\hline Points awarded. & Method & Description \\
\hline \multicolumn{3}{|l|}{ Study design } \\
\hline 3 & Prospective & $\begin{array}{l}\text { Each participants is surveyed before the pandemic and during the } \\
\text { pandemic and their dietary intake compared between time points }\end{array}$ \\
\hline 2 & Pre/post & $\begin{array}{l}\text { Separate groups of participants are surveyed either before or during } \\
\text { the pandemic, and differences in the intake are based on group comparisons }\end{array}$ \\
\hline 1 & Retrospective & $\begin{array}{l}\text { Cross-sectional design in which participants surveyed during the pandemic } \\
\text { are asked to recall their diet before the pandemic and/or comment on changes }\end{array}$ \\
\hline \multicolumn{3}{|c|}{ Dietary assessment method } \\
\hline 7 & Objective assessment & $\begin{array}{l}\text { Dietary intake is either observed by trained study staff or ascertained by a } \\
\text { blood-based nutritional marker }\end{array}$ \\
\hline 6 & Multiple 24-h recall & $\begin{array}{l}\text { Participants are asked to recall their dietary intake for } 24 \mathrm{~h} \text {, in the presence of } \\
\text { trained study staff, for at least one weekend day and one weekday }\end{array}$ \\
\hline 5 & Single $24-\mathrm{h}$ recall/validated FFQ & $\begin{array}{l}\text { Participants are asked to recall their dietary intake for } 24 \mathrm{~h} \text {, for a single } 24-\mathrm{h} \\
\text { period, or participants complete a validated FFQ }\end{array}$ \\
\hline 4 & Validated study-specific questionnaire & $\begin{array}{l}\text { Participants complete a questionnaire developed for the study under question, } \\
\text { which asks about the intake of several foods and has been validated } \\
\text { in previous analyses }\end{array}$ \\
\hline 3 & FFQ & Participants complete an FFQ which has not been previously validated \\
\hline 2 & Study-specific questionnaire & $\begin{array}{l}\text { Participants complete a questionnaire developed for the study under question, } \\
\text { which asks about the intake of several foods which has not been } \\
\text { validated in previous analyses }\end{array}$ \\
\hline 1 & Study-specific single-item question & $\begin{array}{l}\text { Participants are asked a single question about their intake (usually } \\
\text { relating to a single food item) }\end{array}$ \\
\hline
\end{tabular}

Abbreviations: $F F Q$ food frequency questionnaire

chocolate [38], ice-cream [33, 38], calorically dense lownutrient snacks [49], desserts (including pastries) [33, 34, 38, $43,47,50]$, saturated fats [53], and salty snacks [33, 38]. Yet, these findings were also not reported consistently (e.g., there are reports of no change in intake of salty and/or highly caloric snack foods [38], red meat [54], and egg consumption [54]). Changes which were harder to categorize as positive or negative included increases in pasta [47], bread [47], bread-like products [47], and coffee [47].

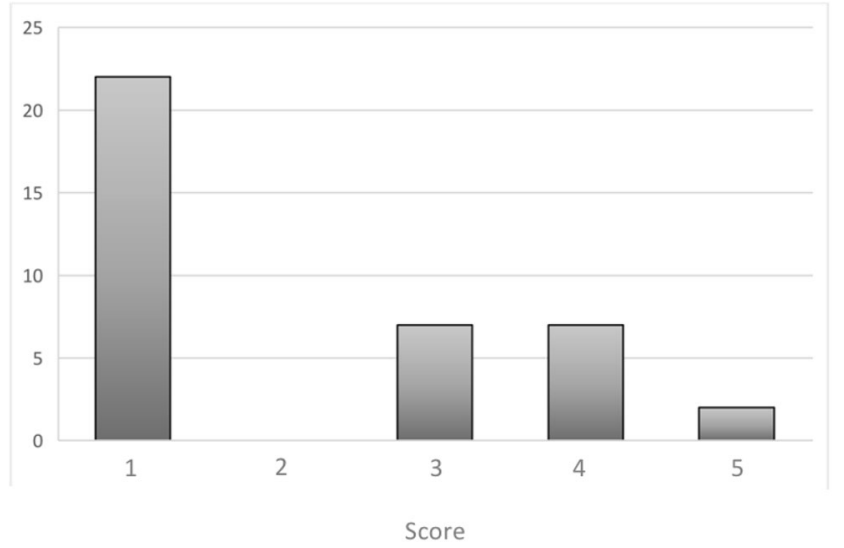

Fig. 2 Distribution of study quality scores

\section{Alcohol}

Whether alcohol intake increased or decreased was similarly hard to categorize. Several studies, even those that may have minimized the tendency to give socially desirable answers by collecting anonymous surveys online, reported a decrease in alcohol consumption $[36,45,51]$. Yet, others report no overall change [52] and some an overall increase [33, 34, 36, 46, 47]. One study found that an overall increase was attributable to male participants only [46].

\section{Children and Adolescents}

It was also difficult to discern a consistent pattern of findings in younger populations. Adolescents were reported to consume more fruit [56], vegetables [56], legumes [56], fried [56], and sweet [56] foods during the pandemic, but these findings in fruit [57••] and vegetables [57••] are disputed. A decrease in meat [56] and SSBs [56] was reported by one study but lacks support from similar findings in other studies. Younger children were reported to consume more potato chips [58], red meat [58], and more sweet, highly caloric snack foods [59], with one study suggesting this latter arose from changes in food parenting practices [59], suggesting their dietary quality may have decreased during the pandemic. 
However, there was also a report of no changes in vegetable intake [58], alongside an increase in fruit intake [58].

\section{Findings in Clinical Populations}

Adults with diabetes represented the majority of clinical samples and reported consuming more carbohydrates [60], fat [60], water [61], fresh fruits and/or vegetables [61-63], dairy [63], and whole grains [61], with notable decreases in salty [61] and sweet [61] snacks in one study, but not in another [63], and decreases in red meat [61]. One study reported that patients with diabetes perceived that their intake of nuts [63], cereal [63], legumes [63], fruit [63], and meat [63], to increase. Pregnant women often reported their overall diet quality did not change $[64,65]$. Although one study supported this notion, reporting no changes in the consumption of individual foods [65], others reported changes indicative of a change in dietary quality. Decreased fish and seafood [64], sugar, and honey [64]; oils and fats [64]; meat [64], poultry [64], offal [64], legumes [64], and of fried food [62] intake; and increased fruits and/or vegetables [64], eggs [64], dairy [64], and cereal [64] intake were reported. However, the extent to which any changes can be attributed to the pandemic environment versus expected changes during pregnancy is not clear.

\section{Synthesis}

Overall, the data do not provide a persuasive picture of whether individuals' dietary intake changed over the course of the pandemic. Perhaps expectedly, it is also impossible to isolate changes in the intake of individual foods which could give clues into changes in overall dietary quality. While the body of literature is impressive for the recency of the pandemic, with the 38 included studies being published within 11 months of the WHO declaration, we found that the studies suffered from an overall low study quality. In addition, children and adolescents are not well represented in the literature.

\section{Part II: An Expert Opinion on how the Pandemic Has Altered the Family Feeding Situation}

\section{Context}

Part I reviewed the existing studies seeking to examine changes in dietary quality occurring over the COVID-19 pandemic. The conflicting results, generally low methodological quality of studies, and paucity of data on children and adolescents precluded conclusions on whether such changes occurred, and if so, what the nature and extent of these were. In the first author's experience as a registered dietitian, who continued to work with families during this time, clients described a clear shift in the psychosocial environment, including shifts in factors which prior research has shown to influence dietary quality and feeding decisions. In Part II of this manuscript, we provide a narrative review in support of the notion that such changes occurred and infer what the effect these may have had on children's dietary quality based the first author's observations and the existing literature.

\section{Historical Context}

As the incidence of two income families continues to grow in the USA, rising from approximately $25 \%$ of the US population in 1960 to approximately $60 \%$ at the turn of the century and subsequently plateauing [66], the likelihood of traditional meals together as a family seems to lessen - an observation tentatively supported by early research [67-74]. Further, the time spent cooking at home is lessened in dual-income families $[70,73]$. What the first author has seen is that between exhausted parents, busy schedules, and isolated family members, a sit-down meal, at home, cooked by one of the parents, is an increasing rarity in this century. Many families are now in their second or third generation of two working parents which, following the Becker/Grossman framework for understanding how increased maternal employment might affect the family as a function of trade-offs in parental time and resources to invest in children [75-79], means many of the current parents who are struggling to provide realistic family meals were also not raised with an expectation of family meals by their own dual working parents. In addition, this shift has coincided with the growth of the wellness industry $[80,81]$ which is recognized as heavily devoted to fad diets [81], supplements [80], and food products that the first author's clients feel offer a "fountain of youth," or "magic lamp," if you will. It seems many marketing schemes promise to make health and wellness easy as long as you use this supplement/ food/ program/new idea to fix all of your wellness problems. The propensity to rely on diet culture and the supplement industry for our wellness information further confuses adults and children alike, therefore perpetuating the problem of discovering balance and pursuing wellness. As we are bombarded with all of this information, it creates nearly impossible decisionmaking for the uneducated consumer. As women have continued to gain traction in the workplace over nearly the last century, they have struggled for equality in benefits, pay, and authority. One area that has consistently lagged behind in women's equality is the responsibility of managing a household and children [82, 83]. As women have continued to work longer hours for (typically) less pay, they are often expected to manage the social calendar, domestic economy, family medical needs, and educational progress of the entire family. It is no secret that this expectation to be sacrificially committed at work and also sacrificially committed to their homes have left many women, mothers in particular, feeling overwhelmed, 
exhausted, and inadequate for decades. In Spring of 2020, a new burden arrived to rest on the shoulders of the overworked mother: COVID-19.

\section{The Psychosocial Environment of the COVID-19 Pandemic}

As the virus has expanded its grasp on our society, the economic effects of COVID-19 have been overwhelming for parents. Parents took on even more responsibilities in the home, like schooling and daily child supervision, in addition to their now remote work. Among the first author's clients, these economic effects have been disproportionately felt more by women than men, especially when children are in the family. And their experience reflects the emerging global data: for example, the productivity of academics dropped more when children were in the home [84-87], with female academics disproportionately affected $\left[84,86,88,89^{\circ}, 90,91\right]$.

Many families entered some kind of quarantine and/or isolation in Spring of 2020 that then continued into 2021. During this quarantine, parents were now performing traditional jobs in a remote work setting and learning while they go. While balancing this unexpected and drastic shift in work that requires multitasking child supervision and adult workload, parents were also expected to learn to facilitate distance education with their children, oftentimes requiring hours on end of screen time in the name of "learning." With work and schooling also came the constant presence of the family in the home, leading to more laundry, more opportunities for food consumption in the home, and more opportunity for conflict with one another. In addition to all of the stressors at home comes a constant barrage of information that parents must filter through to glean the most important and pertinent information for their family and information about the virus, the vaccine, safety protocols, quarantine requirements, political and racial unrest, and an unknown timeline of return to "normalcy."

\section{Anticipated Effects of the Pandemic on Family Dietary Quality}

Even before the pandemic, environmental, financial, and timerelated stressors - particularly as perceived by the motheradversely affected the diet quality of children [92•, 93, 94]. The mental load of COVID isolation has been described in many anecdotal pieces; however, the facts to support the potential unforeseen side effect of these stresses on family dietary intake are only now emerging in recent research. Yet, this mental load of working, parenting, supervising, entertaining, distancing, protecting, and housekeeping fits within popular frameworks regarding the development of decision fatigue $[95,96]$ - and even before the pandemic were considered factors which negatively impacted how parents decided to feed themselves and their families [97-100], termed a "spillover effect" [100]. At the end of a hard day of thinking about all of these things, the idea of making another decision, albeit about the family meal, is exhausting - enough for many of the first-author's clients to give up. In the first author's experience, many families have resorted to take-out meals to not only lessen the decision fatigue but also in an effort to find a reason to leave their house and move about their local area, not to mention support local businesses.

Low-income families, in particular, may find their environment adversely affected by the pandemic. Low-income families often rely on free and reduced school breakfasts and lunches to meet the food needs of their children. However, when schools moved to a virtual and distance learning mode, many families (half of pre-pandemic recipients in one analysis [101]) were robbed of this opportunity to regularly feed their children adequate meals [101-105]. School districts across the country have offered free and reduced school lunches to every student, regardless of income, for the 2020-2021 school year, but these can be difficult to receive for children who are not learning in person.

\section{Conclusions}

The current evidence does not provide evidence, from which persuasive conclusions about any changes in dietary quality over the pandemic can be inferred. It is not yet clear whether the dietary quality of children and adolescents changes, and if this occurred, what the specific foods are where changes in intake might account for changes. Yet, economic, financial, and time-related stressors have increased during the pandemic, and a strong body of literature supports that these stressors negatively impacted the dietary quality of families before the pandemic. This supports the notion that the change in dietary quality of families with young children is distinct from and may show steeper declines when compared with the change in dietary quality socioeconomic, racial, and geographical counterparts without children. Some supportive data are emerging. One study reported an overall decline in alcohol intake during the pandemic, with the exception of families with young children whose home were significantly more likely to report drinking a lot more during the pandemic (10 vs. 5.8\%), while the opposite was true of households without children at home (12 vs. 16.2\%) [106]. Yet, much more high-quality evidence is needed.

Given the pervasive methodological limitations noted in this literature, and the observations of the first-author, it is imperative that the potential impact of the COVID-19 pandemic on the dietary quality of families is not forgotten, nor neglected, but prioritized in research. While the effects of isolation and quarantine from COVID 19 are still being studied, one thing remains certain: feeding families healthy and balanced meals that do not feel like a burden is more difficult than ever before. Studies must examine the extent to which families' dietary quality has been an unanticipated casualty of 
the pandemic and continue to identify strategies to mitigate these effects.

Acknowledgements Dr. Wood's role on this project was funded, in part, by USDA/ARS cooperative agreement \# 58-3092-5-001. The contents of this publication do not necessarily reflect the views or policies of the US Department of Agriculture nor does mention of trade names, commercial products, or organizations imply endorsement by the US Government.

\section{References}

Papers of particular interest, published recently, have been highlighted as:

- Of importance

- Of major importance

1. WHO I. Novel Coronavirus-China. 2020.

2. Organization WH. Listings of WHO's response to COVID-19. WHO [Internet]. 2020.

3. Wasiuta M. Worldometers. In: JSTOR; 2009.

4. Worldometers. Coronavirus. Bulgaria. 2020.

5. PER ADC. COVID Data Tracker.

6. Xiong J, Lipsitz O, Nasri F, et al. Impact of COVID-19 pandemic on mental health in the general population: A systematic review. $\mathrm{J}$ Affect Disord. 2020.

7. Chen H, Guo J, Wang C, et al. Clinical characteristics and intrauterine vertical transmission potential of COVID-19 infection in nine pregnant women: a retrospective review of medical records. Lancet. 2020;395(10226):809-15.

8. Roesch E, Amin A, Gupta J, Garcia-Moreno C. Violence against women during covid-19 pandemic restrictions. BMJ. 2020;369: $\mathrm{m} 1712$.

9. Calderon-Anyosa RJC, Kaufman JS. Impact of COVID-19 lockdown policy on homicide, suicide, and motor vehicle deaths in Peru. Prev Med. 2021;143:106331.

10. McIntyre RS, Lee Y. Projected increases in suicide in Canada as a consequence of COVID-19. Psychiatry Res. 2020;290:113104.

11. John A, Okolie C, Eyles E, et al. The impact of the COVID-19 pandemic on self-harm and suicidal behaviour: a living systematic review. F1000Research. 2020;9.

12. Garcia-Esquinas E, Ortolá R, Gine-Vazquez I, et al. Changes in health behaviors, mental and physical health among older adults under severe lockdown restrictions during the COVID-19 pandemic in Spain. medRxiv. 2021.

13. Elran-Barak R, Mozeikov M. One month into the reinforcement of social distancing due to the COVID-19 outbreak: subjective health, health behaviors, and loneliness among people with chronic medical conditions. Int J Environ Res Public Health. 2020;17(15):5403.

14. Ruiz MC, Devonport TJ, Chen-Wilson C-HJ, et al. A crosscultural exploratory study of health behaviors and wellbeing during COVID-19. Front Psychol. 2020;11.

15. Karbownik MS, Dobielska M, Paul E, Kowalczyk RP, Kowalczyk E. Health-, medication-and dietary supplementrelated behaviors and beliefs relatively unchanged during the COVID-19 pandemic lockdown. Res Soc Adm Pharm. 2020.

16. Brooks SK, Webster RK, Smith LE, et al. The psychological impact of quarantine and how to reduce it: rapid review of the evidence. Lancet. 2020;395(10227):912-20.
17. Sugaya N, Yamamoto T, Suzuki N, Uchiumi C. A real-time survey on the psychological impact of mild lockdown for COVID-19 in the Japanese population. Sci Data. 2020;7(1):372.

18. Salfi F, Lauriola M, Amicucci G, et al. Gender-related time course of sleep disturbances and psychological symptoms during the COVID-19 lockdown: a longitudinal study on the Italian population. Neurobiol Stress. 2020;13:100259.

19. Di Giuseppe M, Zilcha-Mano S, Prout TA, Perry JC, Orru G, Conversano C. Psychological impact of coronavirus disease 2019 among Italians during the first week of lockdown. Front Psychiatry. 2020;11:576597.

20. Burhamah W, AlKhayyat A, Oroszlanyova M, et al. The psychological burden of the COVID-19 pandemic and associated lockdown measures: experience from 4000 participants. J Affect Disord. 2020;277:977-85.

21. Gopal A, Sharma AJ, Subramanyam MA. Dynamics of psychological responses to COVID-19 in India: a longitudinal study. PLoS One. 2020;15(10):e0240650.

22. Yamamoto T, Uchiumi C, Suzuki N, Yoshimoto J, MurilloRodriguez E. The psychological impact of "mild lockdown" in Japan during the COVID-19 pandemic: a nationwide survey under a declared state of emergency. Int J Environ Res Public Health. 2020;17(24).

23. <SSRN-id3675022.pdf > .

24. Santos Machado L, Perlin M, Colla Soletti R, et al. Parent in science: the impact of parenthood on the scientific career in Brazil. 2019 IEEE/ACM 2nd International workshop on gender equality in software engineering (GE); 2019.

25. Power K. The COVID-19 pandemic has increased the care burden of women and families. Sustainability: Science, Practice and Policy. 2020;16(1):67-73.

26. O'neil A, Quirk SE, Housden S, et al. Relationship between diet and mental health in children and adolescents: a systematic review. American Journal of Public Health American Journal of Public Health. 2014;104(10):e31-42.

27. Açik M, Altan M, Cakiroğlu FP. A cross-sectionally analysis of two dietary quality indices and the mental health profile in female adults. Current Psychology. 2020.

28. Hanson KL, Connor LM. Food insecurity and dietary quality in US adults and children: a systematic review. Am J Clin Nutr. 2014;100(2):684-92.

29. Booth A, Clarke M, Dooley G, et al. PROSPERO at one year: an evaluation of its utility. Systematic Reviews. 2013;2(1):1-7.

30. Kleijnen J, Moher D. The PRISMA statement for reporting systematic reviews and meta-analyses of studies that evaluate health care interventions: explanation and elaboration. 2009.

31. Robinson E, Boyland E, Chisholm A, et al. Obesity, eating behavior and physical activity during COVID-19 lockdown: a study of UK adults. Appetite. 2021;156:104853.

32. Cheikh Ismail L, Osaili TM, Mohamad MN, et al. Eating habits and lifestyle during COVID-19 lockdown in the United Arab Emirates: a cross-sectional study. Nutrients. 2020;12(11).

33. Gornicka M, Drywien ME, Zielinska MA, Hamulka J. Dietary and lifestyle changes during COVID-19 and the subsequent lockdowns among Polish adults: a cross-sectional online survey PLifeCOVID-19 study. Nutrients. 2020;12(8).

34. Giacalone D, Frøst MB, Rodríguez-Pérez C. Reported changes in dietary habits during the Covid-19 lockdown in the Danish population: the Danish COVIDiet study. Frontiers in Nutrition. 2020;7:294.

35. Jia $\mathrm{P}, \mathrm{Liu} \mathrm{L}, \mathrm{Xie} \mathrm{X}$, et al. Changes in dietary patterns among youths in China during COVID-19 epidemic: the COVID-19 impact on lifestyle change survey (COINLICS). Appetite. 2021;158:105015.

36. Marty L, de Lauzon-Guillain B, Labesse M, Nicklaus S. Food choice motives and the nutritional quality of diet during the COVID-19 lockdown in France. Appetite. 2021;157:105005. 
37. Poelman MP, Gillebaart M, Schlinkert C, et al. Eating behavior and food purchases during the COVID-19 lockdown: a crosssectional study among adults in the Netherlands. Appetite. 2021;157:105002.

38. Rolland B, Haesebaert F, Zante E, Benyamina A, Haesebaert J, Franck N. Global changes and factors of increase in caloric/salty food intake, screen use, and substance use during the early COVID-19 containment phase in the general population in France: survey study. JMIR Public Health Surveill. 2020;6(3):e19630.

39. Cancello R, Soranna D, Zambra G, Zambon A, Invitti C. Determinants of the lifestyle changes during COVID-19 pandemic in the residents of Northern Italy. Int J Environ Res Public Health. 2020;17(17).

40. Alhusseini N, Alqahtani A. COVID-19 pandemic's impact on eating habits in Saudi Arabia. Journal of Public Health Research. 2020;9(3).

41. Steele EM, Rauber F. Costa CdS, et al. dietary changes in the NutriNet Brasil cohort during the covid-19 pandemic. Rev Saude Publica. 2020;54:91.

42. Rossinot H, Fantin R, Venne J. Behavioral Changes During COVID-19 Confinement in France: a web-based study. Int J Environ Res Public Health. 2020;17(22).

43. Rodriguez-Perez C, Molina-Montes E, Verardo V, et al. Changes in dietary behaviours during the COVID-19 outbreak confinement in the Spanish COVIDiet Study. Nutrients. 2020;12(6).

44. Sanchez-Sanchez E, Ramirez-Vargas G, Avellaneda-Lopez Y, Orellana-Pecino JI, Garcia-Marin E, Diaz-Jimenez J. Eating habits and physical activity of the spanish population during the COVID19 pandemic period. Nutrients. 2020;12(9).

45. Di Renzo L, Gualtieri P, Pivari F, et al. Eating habits and lifestyle changes during COVID-19 lockdown: an Italian survey. J Transl Med. 2020;18(1):229.

46. Ferrante G, Camussi E, Piccinelli C, et al. Did social isolation during the SARS-CoV-2 epidemic have an impact on the lifestyles of citizens? Epidemiol Prev. 2020;44(5-6 Suppl 2):353-62.

47. Cicero AF, Fogacci F, Giovannini M, et al. COVID-19-related quarantine effect on dietary habits in a northern Italian rural population: data from the Brisighella heart study. Nutrients. 2021;13(2):309.

48. Dragun R, Veček NN, Marendić M, et al. Have lifestyle habits and psychological well-being changed among adolescents and medical students due to COVID-19 lockdown in Croatia? Nutrients. 2021;13(1):97.

49. Buckland NJ, Swinnerton LF, Ng K, et al. Susceptibility to increased high energy dense sweet and savoury food intake in response to the COVID-19 lockdown: the role of craving control and acceptance coping strategies. Appetite. 2021;158:105017.

50. Blaszczyk-Bebenek E, Jagielski P, Boleslawska I, Jagielska A, Nitsch-Osuch A, Kawalec P. Nutrition Behaviors in Polish Adults before and during COVID-19 Lockdown. Nutrients. 2020;12(10).

51. Scarmozzino F, Visioli F. Covid-19 and the subsequent lockdown modified dietary habits of almost half the population in an Italian sample. Foods. 2020;9(5).

52. Kriaucioniene V, Bagdonaviciene L, Rodriguez-Perez C, Petkeviciene J. Associations between changes in health behaviours and body weight during the COVID-19 quarantine in Lithuania: The Lithuanian COVIDiet Study. Nutrients. 2020;12(10).

53. Murphy B, Benson T, McCloat A, et al. Changes in consumers' food practices during the COVID-19 lockdown, implications for diet quality and the food system: a cross-continental comparison. Nutrients. 2021;13(1):20.

54. Matsungo TM, Chopera P. Effect of the COVID-19-induced lockdown on nutrition, health and lifestyle patterns among adults in Zimbabwe. BMJ Nutr Prev Health. 2020;3(2):205-12.
55. Sharma SV, Chuang R-J, Rushing M, et al. Peer reviewed: social determinants of health-related needs during COVID-19 among low-income households with children. Prev Chronic Dis. 2020;17.

56. Ruiz-Roso MB, de Carvalho Padilha P, Mantilla-Escalante DC, et al. Covid-19 confinement and changes of adolescent's dietary trends in Italy, Spain, Chile, Colombia and Brazil. Nutrients. 2020;12(6): 1807.

57.• Munasinghe S, Sperandei S, Freebairn L, et al. The impact of physical distancing policies during the COVID-19 pandemic on health and well-being among Australian adolescents. J Adolesc Health. 2020;67(5):653-61 The only prospective study on pandemic-related dietary changes, which inlcudes dietary intake measured using a validated questinnaire on individuals under 18 years of age.

58. Pietrobelli A, Pecoraro L, Ferruzzi A, et al. Effects of COVID-19 lockdown on lifestyle behaviors in children with obesity living in Verona, Italy: a longitudinal study. Obesity (Silver Spring). 2020;28(8):1382-5.

59. Jansen E, Thapaliya G, Aghababian A, Sadler J, Smith K, Carnell S. Parental stress, food parenting practices and child snack intake during the COVID-19 pandemic. Appetite 105119.

60. Ghosh A, Arora B, Gupta R, Anoop S, Misra A. Effects of nationwide lockdown during COVID-19 epidemic on lifestyle and other medical issues of patients with type 2 diabetes in North India. Diabetes Metab Syndr. 2020;14(5):917-20.

61. Grabia M, Markiewicz-Zukowska R, Puscion-Jakubik A, et al. The nutritional and health effects of the COVID-19 pandemic on patients with diabetes mellitus. Nutrients. 2020;12(10).

62. Sankar P, Ahmed WN, Mariam Koshy V, Jacob R, Sasidharan S. Effects of COVID-19 lockdown on type 2 diabetes, lifestyle and psychosocial health: a hospital-based cross-sectional survey from South India. Diabetes Metab Syndr. 2020;14(6):1815-9.

63. Ruiz-Roso MB, Knott-Torcal C, Matilla-Escalante DC, et al. COVID-19 lockdown and changes of the dietary pattern and physical activity habits in a cohort of patients with type 2 diabetes mellitus. Nutrients. 2020;12(8):2327.

64. Zhang J, Zhang Y, Huo S, et al. Emotional eating in pregnant women during the COVID-19 pandemic and its association with dietary intake and gestational weight gain. Nutrients. 2020;12(8).

65. Bivia-Roig G, La Rosa VL, Gomez-Tebar M, et al. Analysis of the impact of the confinement resulting from COVID-19 on the lifestyle and psychological wellbeing of Spanish pregnant women: an internet-based cross-sectional survey. Int J Environ Res Public Health. 2020;17(16).

66. Center PR. The Rise in Dual Income Families. 2015.

67. Anderson PM. Parental employment, family routines and childhood obesity. Economics \& Human Biology. 2012;10(4):340-51.

68. Allen TD, Shockley KM, Poteat LF. Workplace factors associated with family dinner behaviors. J Vocat Behav. 2008;73(2):336-42.

69. Neumark-Sztainer D, Hannan PJ, Story M, Croll J, Perry C. Family meal patterns: associations with sociodemographic characteristics and improved dietary intake among adolescents. J Am Diet Assoc. 2003;103(3):317-22.

70. Cawley J, Liu F. Maternal employment and childhood obesity: a search for mechanisms in time use data. Economics \& Human Biology. 2012;10(4):352-64.

71. Möser A, Chen SE, Jilcott SB, Nayga RM. Associations between maternal employment and time spent in nutrition-related behaviours among German children and mothers. Public Health Nutr. 2012;15(7):1256-61.

72. Sharif MZ, Alcalá HE, Albert SL, Fischer H. Deconstructing family meals: do family structure, gender and employment status influence the odds of having a family meal? Appetite. 2017;114: 187-93.

73. Spear BA. The need for family meals. J Am Diet Assoc. 2006;106(2):218-9. 
74. Fertig A, Glomm G, Tchernis R. The connection between maternal employment and childhood obesity: inspecting the mechanisms. Rev Econ Househ. 2009; 7(3):227-55.

75. Becker GS, Lewis HG. On the interaction between the quantity and quality of children. J Polit Econ. 1973;81(2, Part 2):S279-88.

76. Grossman M. On the concept of health capital and the demand for health. J Polit Econ. 1972;80(2):223-55.

77. Bianchi SM. Maternal employment and time with children: dramatic change or surprising continuity? Demography. 2000;37(4): $401-14$.

78. London AS, Scott EK, Edin K, Hunter V. Welfare reform, workfamily tradeoffs, and child well-being. Fam Relat. 2004;53(2): $148-58$.

79. Scott EK, Edin K, London AS, Kissane RJ. Unstable work, unstable income: implications for family well-being in the era of time-limited welfare. J Poverty. 2004;8(1):61-88.

80. Kickbusch I, Payne L. Twenty-first century health promotion: the public health revolution meets the wellness revolution. In: Oxford University Press; 2003.

81. Rousseau S. The celebrity quick-fix: when good food meets bad science. Food, Culture \& Society. 2015;18(2):265-87.

82. Easterbrooks M, Goldberg WA. Effects of early maternal employment on toddlers, mothers, and fathers. Dev Psychol. 1985;21(5): 774.

83. Pedersen FA, Cain RL, Zaslow MJ, Anderson BJ. Variation in infant experience associated with alternative family roles. In: Families as learning environments for children. Springer; 1982: 203-221.

84. Krukowski RA, Jagsi R, Cardel MI. Academic productivity differences by gender and child age in science, technology, engineering, mathematics, and medicine faculty during the COVID-19 pandemic. J Women's Health. 2020.

85. Chapman DG, Thamrin C. Scientists in pyjamas: characterising the working arrangements and productivity of Australian medical researchers during the COVID-19 pandemic. Med J Aust. 2020;213(11):516-20.

86. Staniscuaski F, Kmetzsch L, Zandona E, et al. Gender, race and parenthood impact academic productivity during the COVID-19 pandemic: from survey to action. bioRxiv. 2020.

87. Myers KR, Tham WY, Yin Y, et al. Unequal effects of the COVID-19 pandemic on scientists. Nat Hum Behav. 2020;4(9): $880-3$.

88. Cui R, Ding H, Zhu F. Gender inequality in research productivity during the COVID-19 pandemic. arXiv preprint arXiv: 200610194. 2020.

89. Feng Z, Savani K. Covid-19 created a gender gap in perceived work productivity and job satisfaction: implications for dualcareer parents working from home. Gender in Management: An International Journal. 2020. Descriptive study on psychosocial changes occurring during the pandemic, including an assessment of gender-specific effects.

90. King MM, Frederickson M. The Pandemic Penalty: The gendered effects of COVID-19 on scientific productivity. 2020.

91. Andersen JP, Nielsen MW, Simone NL, Lewiss RE, Jagsi R. Meta-research: COVID-19 medical papers have fewer women first authors than expected. Elife. 2020;9:e58807.

92. Beshara M, Hutchinson A, Wilson C. Preparing meals under time stress. The experience of working mothers. Appetite. 2010;55(3): 695-700 Provides strong empirical support for the effects of maternal stress on children's dietary intake.

93. Warren EJ, Font SA. Housing insecurity, maternal stress, and child maltreatment: an application of the family stress model. Soc Serv Rev. 2015;89(1):9-39.
94. Trude AC, Black MM, Surkan PJ, Hurley KM, Wang Y. Maternal anxiety and diet quality among mothers and toddlers from lowincome households. Maternal \& child nutrition. 2020;16(4): e12992.

95. Pignatiello GA, Martin RJ, Hickman RL Jr. Decision fatigue: a conceptual analysis. J Health Psychol. 2020;25(1):123-35.

96. Vohs KD, Baumeister RF, Twenge JM, Schmeichel BJ, Tice DM, Crocker J. Decision fatigue exhausts self-regulatory resourcesBut so does accommodating to unchosen alternatives. Manuscript submitted for publication. 2005.

97. Brophy-Herb HE, Horodynski M, Contreras D, et al. Effectiveness of differing levels of support for family meals on obesity prevention among head start preschoolers: the simply dinner study. BMC Public Health. 2017;17(1):1-11.

98. Blake CE, Devine CM, Wethington E, Jastran M, Farrell TJ, Bisogni CA. Employed parents' satisfaction with food-choice coping strategies. Influence of gender and structure. Appetite. 2009;52(3):711-9.

99. Jarrett RL, Bahar OS, Kersh RT. "When we do sit down together" family meal times in low-income African American families with preschoolers. J Fam Issues. 2016;37(11):1483-513.

100. Devine CM, Stoddard AM, Barbeau EM, Naishadham D, Sorensen G. Work-to-family spillover and fruit and vegetable consumption among construction laborers. Am J Health Promot. 2007;21(3):175-82.

101. Parnham JC, Laverty AA, Majeed A, Vamos EP. Half of children entitled to free school meals did not have access to the scheme during COVID-19 lockdown in the UK. Public Health. 2020;187: $161-4$.

102. Dunn CG, Kenney E, Fleischhacker SE, Bleich SN. Feeding lowincome children during the Covid-19 pandemic. N Engl J Med. 2020;382(18):e40.

103. Mayurasakorn K, Pinsawas B, Mongkolsucharitkul P, Sranacharoenpong K. Damapong Sn. School closure, COVID19 and lunch programme: unprecedented undernutrition crisis in low-middle income countries. J Paediatr Child Health. 2020;56(7):1013-7.

104. Kinsey EW, Hecht AA, Dunn CG, et al. School closures during COVID-19: opportunities for innovation in meal service. Am J Public Health. 2020;110(11):1635-43.

105. Kinsey EW, Kinsey D, Rundle AG. COVID-19 and food insecurity: an uneven patchwork of responses. J Urban Health. 2020;97: 332-5.

106. Ingram J, Maciejewski G, Hand CJ. Changes in diet, sleep, and physical activity are associated with differences in negative mood during COVID-19 lockdown. Front Psychol. 2020;11:588604.

107. Romero-Blanco C, Rodriguez-Almagro J, Onieva-Zafra MD, Parra-Fernandez ML, Prado-Laguna MDC, Hernandez-Martinez A. Physical Activity and Sedentary Lifestyle in University Students: Changes during Confinement Due to the COVID-19 Pandemic. Int J Environ Res Public Health. 2020;17(18).

108. Zhang J, Zhao A, Ke Y, et al. Dietary behaviors in the postlockdown period and its effects on dietary diversity: the second stage of a nutrition survey in a longitudinal Chinese study in the COVID-19 Era. Nutrients. 2020;12(11).

109. Bin Zarah A, Enriquez-Marulanda J, Andrade JM. Relationship between dietary habits, food attitudes and food security status among adults living within the United States three months postmandated quarantine: a cross-sectional study. Nutrients. 2020;12(11):3468.

Publisher's Note Springer Nature remains neutral with regard to jurisdictional claims in published maps and institutional affiliations. 